\title{
GEOMETRY OF POINTWISE SEMI-SLANT WARPED PRODUCTS IN LOCALLY CONFORMAL KAEHLER MANIFOLDS
}

\author{
BANG-YEN CHEN, FATIMAH ALGHAMDI, AND SIRAJ UDDIN
}

\begin{abstract}
In this paper, we study the geometry of pointwise semi-slant warped products in a locally conformal Kaehler manifold. In particular, we obtain several results which extend Chen's inequality for $C R$-warped product submanifolds in Kaehler manifolds. Also, we study the corresponding equality cases. Several related results on pointwise semi-slant warped products are also proved in this paper.
\end{abstract}

\section{INTRODUCTION}

The notion of slant submanifolds of an almost Hermitian manifold were introduced by first author in 11, 12, which include both totally real and holomorphic submanifolds. Since then many papers on these submanifolds have been published (see, e.g., 20, 21]). Also, as the generalizations of totally real and holomorphic submanifolds, A. Bejancu introduced the notion of CR-submanifolds in [2]. CRsubmanifolds have also been studied by many geometers (see, e.g. 3, 4, 6, 9, 10).

As a generalization of slant submanifolds, N. Papaghiuc 30 introduced the notion of semi-slant submanifolds of an almost Hermitian manifold which includes the classes of CR-submanifolds and slant submanifolds. As another extension of slant submanifolds, F. Etayo [25] defined the notion of pointwise slant submanifolds of almost Hermitian manifolds under the name of quasi-slant submanifolds. Then, the first author and O. J. Garay studied in 19 pointwise slant submanifolds of Kaehler manifolds and they proved several fundamental results of such submanifolds and provided a method to construct examples of such submanifolds.

In the 1960s, R.L. Bishop and B. O'Neill defined warped product manifolds. It is well-known that warped product manifolds play important role in differential geometry as well as in physics. At the beginning of this century, the first author initiated in [13, 14, 15] the study of warped products in Riemannian and Kaehler manifolds from the submanifold point of view. He proved in 13 that there do not exist warped products of the form: $N^{\perp} \times_{f} N^{T}$ in any Kaehler manifold beside $C R$-products, where $N^{\perp}$ is a totally real submanifold and $N^{T}$ is a holomorphic submanifold. He also shown in 13 that there exist many $C R$-submanifolds which are warped products of the form $N^{T} \times{ }_{f} N^{\perp}$ by reversing the two factors $N^{T}$ and $N_{\perp}$. He simply called such $C R$-submanifold a $C R$-warped product. Furthermore, he

2010 Mathematics Subject Classification. 53C15, 53C40, 53C42, 53B25.

Key words and phrases. Warped products; CR-warped product; pointwise semi-slant warped products, locally conformal Kaehler manifold. 
proved that every $C R$-warped product $N^{T} \times_{f} N^{\perp}$ in any Kaehler manifold satisfies the basic inequality:

$$
\|h\|^{2} \geq 2 p\|\vec{\nabla}(\ln f)\|^{2},
$$

where $f$ is the warping function, $p$ is the dimension of $N^{\perp},\|h\|^{2}$ the squared norm of the second fundamental form, and $\vec{\nabla}(\ln f)$ the gradient of $\ln f$. Since then the geometry of warped product submanifolds becomes an active research subject (for more details, we refer to Chen's books [16, 18, and his survey article [17]).

In 31, B. Sahin proved that there do not exist semi-slant warped products in a Kaehler manifold. Later, he investigated warped product pointwise semislant submanifolds of Kaehler manifolds in 32. Further, V, Bonanzinga and K. Matsumoto [7] studied $C R$-warped product submanifolds and semi-slant warped product submanifolds in locally conformal Kaehler manifolds either of the form $N^{T} \times{ }_{f} N^{\perp}$ and or of $N^{T} \times{ }_{f} N^{\theta}$, where $N^{T}, N^{\perp}$ and $N^{\theta}$ are holomorphic, totally real and slant submanifolds, respectively (see also [29]). Since then, such warped product submanifolds have been studied by many geometers (see, e.g., 1, 18]).

In this paper, we investigate pointwise semi-slant warped products in locally conformal Kaehler manifolds. In particular, we obtain several results which extend Chen's inequality (1.1); and we also study the corresponding equality cases. Several related results are also established in this paper.

\section{Preliminaries}

A locally conformally Kaehler manifold $(\tilde{M}, J, g)$ of (or an $L C K$-manifold for short) is a complex manifold $(\tilde{M}, J)$ endowed with a Hermitian metric $g$ which is locally conformal to a Kaehlerian metric. Equivalently, there exists an open cover $\left\{U_{i}\right\}_{i \in I}$ of $\tilde{M}$ and a family $\left\{f_{i}\right\}_{i \in I}$ of real-valued differentiable functions $f_{i}: U_{i} \rightarrow \mathbb{R}$ such that $g_{i}=\left.e^{-f_{i}} g\right|_{U_{i}}$ is a Kaehlerian metric on $U_{i}$, i.e., $\nabla^{*} J=0$, where $J$ is the almost complex structure, $g$ is the Hermitian metric, and $\nabla^{*}$ is the covariant differentiation with respect to $g$. A typical example of a compact $L C K$-manifold is a Hopf manifold which is diffeomorphic to $\mathbb{S}^{1} \times \mathbb{S}^{2 n-1}$ and it admits no Kaehler structure (see 38). Let $\Omega$ and $\Omega_{i}$ denote the 2 -forms associated with $(J, g)$ and $\left(J, g_{i}\right)$, respectively (i.e., $\Omega(X, Y)=g(X, J Y)$, etc.). Then $\Omega_{i}=\left.e^{-f_{i}} \Omega\right|_{U_{i}}$.

The following result from [38 is well-known (see also [24, Theorem 1.1]).

Theorem 2.1. The Hermitian manifold $(\tilde{M}, J, g)$ is an LCK-manifold if and only if there exists a globally defined closed 1 -form $\alpha$ on $\tilde{M}$ such that $d \Omega=\alpha \wedge \Omega$.

The closed 1-form $\alpha$ in Theorem 2.1 is called the Lee form and the vector field $\lambda=\alpha^{\#}$ dual to $\alpha$, (i.e., $g(X, \lambda)=\alpha(X)$ for $X \in T \tilde{M}$ ), is called the Lee vector field of the $L C K$-manifold $\tilde{M}$. An $L C K$-manifold $(\tilde{M}, J, g)$ is called a globally conformal Kaehler manifold (GCK-manifold for short) if one can choose $U=\tilde{M}$. An $L C K$-manifold $\tilde{M}$ is a $G C K$-manifold if and only if the 1-form $\alpha$ is exact.

If $\tilde{\nabla}$ denotes the Levi-Civita connection on an $L C K$-manifold $\tilde{M}$, then we have

$$
\left(\tilde{\nabla}_{X} J\right) Y=-g\left(\beta^{\#}, Y\right) X-g\left(\alpha^{\#}, Y\right) J X+g(J X, Y) \alpha^{\#}+g(X, Y) \beta^{\#} .
$$

for tangent vector fields $X, Y$ on $\tilde{M}$, where $\alpha^{\#}$ is the Lee vector field, $\beta$ is the 1-form defined by $\beta(X)=-\alpha(J X)$ for any $X \in T \tilde{M}$ and $\beta^{\#}$ denotes the dual 
vector field of $\beta$ (see [38). In terms of the Lee vector field, equation (2.1) can be written as

$$
\left.\tilde{\left(\nabla_{X}\right.} J\right) Y=[g(\lambda, J Y) X-g(\lambda, Y) J X+g(J X, Y) \lambda+g(X, Y) J \lambda] .
$$

$L C K$-manifolds contain rich source since their Lee-form plays important role in determining several geometric features of their submanifolds. An $L C K$-manifold $(\tilde{M}, J, g)$ is called a Vaisman manifold if its Lee form is parallel, i.e., $\tilde{\nabla} \alpha=0$ (see [38, 39]). Vaisman manifolds form the most important subclass of $L C K$-manifolds.

Let $(\tilde{M}, J, g, \alpha)$ be a complex $m$-dimensional $L C K$-manifold and $M$ be a real $n$-dimensional Riemannian manifold isometrically immersed in $\tilde{M}$ with $n \leq m$. Denote by $\Gamma(T M)$ and $\Gamma\left(T^{\perp} M\right)$ the spaces of tangent and normal vector fields of $M$, respectively. Let $g$ also denote the induced metric tensor on $M$ and by $\nabla$ the covariant differentiation with respect to the induced metric on $M$. Then the Gauss and Weingarten formulas for $M$ are given respectively by

$$
\begin{aligned}
& \tilde{\nabla}_{X} Y=\nabla_{X} Y+h(X, Y), \\
& \tilde{\nabla}_{X} \xi=-A_{\xi} X+\nabla_{X}^{\perp} \xi,
\end{aligned}
$$

for any $X, Y \in \Gamma(T M)$ and $\xi \in \Gamma\left(T^{\perp} M\right)$, where $\nabla^{\perp}$ is the normal connection, $h$ is the second fundamental form, and $A_{\xi}$ is the Weingarten map (or the shape operator) associated with $\xi$. It is well-known that $A_{\xi}$ and $h$ are related by

$$
g(h(X, Y), \xi)=g\left(A_{\xi} X, Y\right) .
$$

A submanifold $M$ is said to be totally geodesic if its second fundamental form $h$ vanishes identically, i.e., $h=0$, or equivalently $A=0$.

For any vector $X$ tangent to $M$, we write

$$
J X=P X+F X,
$$

where $P X$ and $F X$ are the tangential and normal components of $J X$, respectively. Similarly, for any vector $\xi$ normal to $M$, we put

$$
J \xi=t \xi+f \xi,
$$

where $t \xi$ and $f \xi$ are the tangential and normal components of $J \xi$, respectively.

It was known in [19] that a submanifold $M$ of an almost Hermitian manifold $\tilde{M}$ is pointwise slant if and only if

$$
P^{2}=-\left(\cos ^{2} \theta\right) I,
$$

for some real-valued function $\theta$ defined on $M$, where $I$ is the identity map of the tangent bundle $T M$ of $M$. A pointwise slant submanifold is called proper if it does not contain any totally real or complex points, i.e., $0<\cos ^{2} \theta<1$.

At a given point $p \in M$, the following relations are easy consequences of (2.8):

$$
\begin{gathered}
g(P X, P Y)=\left(\cos ^{2} \theta\right) g(X, Y), \\
g(F X, F Y)=\left(\sin ^{2} \theta\right) g(X, Y)
\end{gathered}
$$

for any $X, Y \in \Gamma(T M)$. Further, it is easy to verify that

$$
t F X=-\sin ^{2} \theta X, \quad f F X=-F P X
$$

for any $X \in \Gamma(T M)$. 
Definition 2.1. Let $\tilde{M}$ be an almost Hermitian manifold and $M$ be a submanifold of $\tilde{M}$. Then $M$ is called a pointwise semi-slant submanifold if there exists a pair of orthogonal distributions $\mathfrak{D}$ and $\mathfrak{D}^{\theta}$ on $M$ such that

(i) The tangent bundle $T M$ is the orthogonal decomposition $T M=\mathfrak{D} \oplus \mathfrak{D}^{\theta}$.

(ii) The distribution $\mathfrak{D}$ is $J$-invariant (or holomorphic), i.e., $J(\mathfrak{D})=\mathfrak{D}$.

(iii) The distribution $\mathfrak{D}^{\theta}$ is pointwise slant with slant function $\theta$.

The pointwise semi-slant submanifold $M$ is called proper if neither $\operatorname{dim} N^{T}=0$ nor the slant function of $\mathfrak{D}^{\theta}$ is $\frac{\pi}{2}$, i.e., $\cos ^{2} \theta>0$. Otherwise, $M$ is called improper.

Definition 2.2. Let $M$ be a submanifold of an almost Hermitian manifold. Then

(i) $M$ is called $\mathfrak{D}$-geodesic if $h(X, Y)=0$,

(ii) $M$ is called $\mathfrak{D}^{\theta}$-geodesic if $h(Z, W)=0$,

(iii) $M$ is called mixed totally geodesic if $h(X, Z)=0$, for all $X, Y \in \Gamma(\mathfrak{D})$ and for all $Z, W \in \Gamma\left(\mathfrak{D}^{\theta}\right)$.

\section{SOME LEMMAS}

First, we prove the following results for later use.

Lemma 3.1. Let $M$ be a proper pointwise semi-slant submanifold of an $L C K$ manifold. Then, for any $X, Y \in \Gamma(\mathfrak{D})$ and $Z \in \Gamma\left(\mathfrak{D}^{\theta}\right)$, we have

$$
\begin{aligned}
\sin ^{2} \theta g\left(\nabla_{X} Y, Z\right)= & g\left(A_{F Z} J Y-A_{F P Z} Y, X\right)-g(J X, Y) g\left(\alpha^{\#}, F Z\right) \\
& -g(X, Y) g\left(\beta^{\#}, F Z\right) .
\end{aligned}
$$

Proof. For any $X, Y \in \Gamma(\mathfrak{D})$ and $Z \in \Gamma\left(\mathfrak{D}^{\theta}\right)$, we have

$$
g\left(\nabla_{X} Y, Z\right)=g\left(J \tilde{\nabla}_{X} Y, J Z\right)=g\left(J \tilde{\nabla}_{X} Y, F Z\right)+g\left(J \tilde{\nabla}_{X} Y, P Z\right) .
$$

From the covariant derivative formula of $J$, we derive

$$
g\left(\nabla_{X} Y, Z\right)=g\left(\tilde{\nabla}_{X} J Y, F Z\right)-g\left(\left(\tilde{\nabla}_{X} J\right) Y, F Z\right)-g\left(\tilde{\nabla}_{X} Y, P^{2} Z\right)-g\left(\tilde{\nabla}_{X} Y, F P Z\right) .
$$

Then, using (2.1), (2.5) and (2.8), we arrive at

$$
\begin{aligned}
g\left(\nabla_{X} Y, Z\right)= & g\left(A_{F Z} J Y, X\right)-g(J X, Y) g(\lambda, F Z)-g(X, Y) g(J \lambda, F Z) \\
& -g\left(Y, \tilde{\nabla}_{X} \cos ^{2} \theta Z\right)-g\left(A_{F P Z} Y, X\right) .
\end{aligned}
$$

Since $M$ is a proper pointwise semi-slant submanifold ,we get

$$
\begin{aligned}
g\left(\nabla_{X} Y, Z\right)= & g\left(A_{F Z} J Y-A_{F P Z} Y, X\right)-g(J X, Y) g(\lambda, F Z)-g(X, Y) g(J \lambda, F Z) \\
& -\cos ^{2} \theta g\left(\tilde{\nabla}_{X} Z, Y\right)+\sin 2 \theta X(\theta) g(Y, Z) .
\end{aligned}
$$

Thus, the lemma follows from above relations by using the orthogonality of the two distributions.

If $\mathfrak{D}$ is a totally geodesic distribution in $M$, then $g\left(\nabla_{X} Y, Z\right)=0$ for any $X, Y \in$ $\Gamma(\mathfrak{D})$ and $Z \in \Gamma\left(\mathfrak{D}^{\theta}\right)$. Hence, Lemma 3.1 implies the following.

Lemma 3.2. Let $M$ be a proper pointwise semi-slant submanifold of an LCKmanifold $\tilde{M}$. Then the distribution $\mathfrak{D}$ defines a totally geodesic foliation if and only if

$$
g\left(A_{F Z} J X-A_{F P Z} X, Y\right)=g(X, Y) g\left(\beta^{\#}, F Z\right)-g(J X, Y) g\left(\alpha^{\#}, F Z\right),
$$


for any $X, Y \in \Gamma(\mathfrak{D})$ and $Z \in \Gamma\left(\mathfrak{D}^{\theta}\right)$.

Corollary 3.1. Let $M$ be a proper pointwise semi-slant submanifold of an LCKmanifold $\tilde{M}$. Then the distribution $\mathfrak{D}$ defines a totally geodesic foliation if and only if

$$
A_{F Z} J X-A_{F P Z} X=g\left(\beta^{\#}, F Z\right) X-g\left(\alpha^{\#}, F Z\right) J X,
$$

for any $X \in \Gamma(\mathfrak{D})$ and $Z \in \Gamma\left(\mathfrak{D}^{\theta}\right)$.

For leaves of the pointwise slant distribution $\mathfrak{D}^{\theta}$, we have the following result.

Lemma 3.3. Let $M$ be a pointwise semi-slant submanifold of an LCK-manifold $\tilde{M}$ with proper pointwise slant distribution $\mathfrak{D}^{\theta}$. Then we have

$$
g\left(\nabla_{Z} W, X\right)=\csc ^{2} \theta g\left(A_{F P W} X-A_{F W} J X, Z\right)-g(Z, W) g\left(\alpha^{\#}, X\right),
$$

for any $X \in \Gamma(\mathfrak{D})$ and $Z, W \in \Gamma\left(\mathfrak{D}^{\theta}\right)$.

Proof. For any for any $X \in \Gamma(\mathfrak{D})$ and $Z, W \in \Gamma\left(\mathfrak{D}^{\theta}\right)$, we find

$$
g\left(\nabla_{Z} W, X\right)=g\left(J \tilde{\nabla}_{Z} W, J X\right)=g\left(\tilde{\nabla}_{Z} J W, J X\right)-g\left(\left(\tilde{\nabla}_{Z} J\right) W, J X\right) .
$$

Using (2.2), we get

$$
\begin{aligned}
g\left(\nabla_{Z} W, X\right)= & g\left(\tilde{\nabla}_{Z} P W, J X\right)+g\left(\tilde{\nabla}_{Z} F W, J X\right)-g(J Z, W) g(\lambda, J X) \\
& -g(Z, W) g(J \lambda, J X) .
\end{aligned}
$$

Then, we derive

$$
\begin{aligned}
g\left(\nabla_{Z} W, X\right)= & -g\left(J \tilde{\nabla}_{Z} P W, X\right)-g\left(A_{F W} Z, J X\right)-g(P Z, W) g(\lambda, J X) \\
& -g(Z, W) g(\lambda, X) .
\end{aligned}
$$

From the definition of covariant derivative of $J$ and the symmetry of the shape operator, we obtain

$$
\begin{aligned}
g\left(\nabla_{Z} W, X\right)= & g\left(\left(\tilde{\nabla}_{Z} J\right) P W, X\right)-g\left(\tilde{\nabla}_{Z} J P W, X\right)-g\left(A_{F W} J X, Z\right) \\
& -g(P Z, W) g(\lambda, J X)-g(Z, W) g(\lambda, X) .
\end{aligned}
$$

Again using (2.2) and (2.6), we derive

$$
\begin{aligned}
g\left(\nabla_{Z} W, X\right)= & g(P Z, P W) g(\lambda, X)+g(Z, P W) g(J \lambda, X)-g\left(\tilde{\nabla}_{Z} P^{2} W, X\right) \\
& -g\left(\tilde{\nabla}_{Z} F P W, X\right)-g\left(A_{F W} J X, Z\right)-g(P Z, W) g(\lambda, J X) \\
& -g(Z, W) g(\lambda, X) .
\end{aligned}
$$

From the relation (2.8), we find

$$
\begin{aligned}
g\left(\nabla_{Z} W, X\right)= & \cos ^{2} \theta g\left(\tilde{\nabla}_{Z} W, X\right)-\sin 2 \theta X(\theta) g(W, X)+g\left(A_{F P W} Z, X\right) \\
& +\cos ^{2} \theta g(Z, W) g(\lambda, X)-g\left(A_{F W} J X, Z\right)-g(Z, W) g(\lambda, X) .
\end{aligned}
$$

By the orthogonality of two distributions, we derive

$$
g\left(\nabla_{Z} W, X\right)=\csc ^{2} \theta\left[g\left(A_{F P W} X, Z\right)-g\left(A_{F W} J X, Z\right)\right]-g(Z, W) g(\lambda, X),
$$

which proves the lemma completely.

Lemma 3.3 implies the following result. 
Corollary 3.2. Let $M$ be a proper pointwise semi-slant submanifold of an $L C K$ manifold $\tilde{M}$. Then the slant distribution $\mathfrak{D}^{\theta}$ defines a totally geodesic foliation if and only if

$$
g\left(A_{F P Z} X-A_{F Z} J X, W\right)=\sin ^{2} \theta g\left(\alpha^{\#}, X\right) g(Z, W)
$$

for any $X \in \Gamma(\mathfrak{D})$ and $Z, W \in \Gamma\left(\mathfrak{D}^{\theta}\right)$.

Lemma 3.4. Let $M$ be a proper pointwise semi-slant submanifold of an LCKmanifold $\tilde{M}$. Then we have

$$
\sin ^{2} \theta g([Z, W], X)=g\left(A_{F Z} J X-A_{F P Z} X, W\right)-g\left(A_{F W} J X-A_{F P W} X, Z\right)
$$

for any $X \in \Gamma(\mathfrak{D})$ and $Z, W \in \Gamma\left(\mathfrak{D}^{\theta}\right)$.

Proof. From Lemma 3.3, we have

$$
\sin ^{2} \theta g\left(\tilde{\nabla}_{Z} W, X\right)=g\left(A_{F P W} X-A_{F W} J X, Z\right)-\sin ^{2} \theta g(\lambda, X) g(Z, W)
$$

for any $X \in \Gamma(\mathfrak{D})$ and $Z, W \in \Gamma\left(\mathfrak{D}^{\theta}\right)$. By interchanging $Z$ and $W$ in (3.1), we find

$$
\sin ^{2} \theta g\left(\tilde{\nabla}_{W} Z, X\right)=g\left(A_{F P Z} X-A_{F Z} J X, W\right)-\sin ^{2} \theta g(\lambda, X) g(Z, W) .
$$

Thus, after subtracting (3.1) from (3.2), we get the required result.

Now, we give the following integrability theorem.

Theorem 3.1. Let $M$ be a pointwise semi-slant submanifold of an LCK-manifold $(\tilde{M}, J, g)$. Then we have

(i) The holomorphic distribution $\mathfrak{D}$ of $M$ is integrable if and only if

$$
h(J Y, X)-h(J X, Y)=2 g(J X, Y) \alpha^{\#}, \forall X, Y \in \Gamma(\mathfrak{D}) .
$$

(ii) The pointwise slant distribution $\mathfrak{D}^{\theta}$ of $M$ is integrable if and only if

$g\left(A_{F W} J X-A_{F P W} X, Z\right)=g\left(A_{F Z} J X-A_{F P Z} X, W\right), \forall X \in \Gamma(\mathfrak{D}), Z, W \in \Gamma\left(\mathfrak{D}^{\theta}\right)$.

Proof. Let $M$ be a proper pointwise semi-slant submanifold of an $L C K$-manifold. Then, by interchanging $X$ and $Y$ and using symmetry of $A$, we find from Lemma 3.1 that

$$
\sin ^{2} \theta g([X, Y], Z)=g\left(A_{F Z} J Y, X\right)-g\left(A_{F Z} J X, Y\right)-2 g(J X, Y) g(\lambda, F Z)
$$

for any $X \in \Gamma(\mathfrak{D})$ and $Z \in \Gamma\left(\mathfrak{D}^{\theta}\right)$. Thus the distribution $\mathfrak{D}$ is integrable if and only if $g([X, Y], Z)=0$ for all $X \in \Gamma(\mathfrak{D})$ and $Z \in \Gamma\left(\mathfrak{D}^{\theta}\right)$, i.e.,

$$
g(h(J Y, X), F Z)-g(h(J X, Y), F Z)=2 g(J X, Y) g(\lambda, F Z) .
$$

Hence, (i) follows from the last relation. In a similar way, we can prove (ii).

\section{Pointwise Semi-Slant Warped Products: $N^{T} \times_{f} N^{\theta}$}

Let $N_{1}$ and $N_{2}$ be two Riemannian manifolds with Riemannian metrics $g_{1}$ and $g_{2}$, respectively, and $f$ be a positive differential function on $N_{1}$. Consider the product manifold $N_{1} \times N_{2}$ with its natural projections $\pi_{1}: N_{1} \times N_{2} \rightarrow N_{1}$ and $\pi_{2}: N_{1} \times N_{2} \rightarrow N_{2}$. Then the warped product manifold $N_{1} \times{ }_{f} N_{2}$ is the product manifold $N_{1} \times N_{2}$ equipped with the warped product metric $g$ defined by

$$
g(X, Y)=g_{1}\left(\pi_{1 \star} X, \pi_{1 \star} Y\right)+\left(f \circ \pi_{1}\right)^{2} g_{2}\left(\pi_{2 \star} X, \pi_{2 \star} Y\right)
$$


for $X, Y \in \Gamma(T M)$, where $\pi_{i \star}$ is the tangent map of $\pi_{i}$. The function $f$ is called the warping function on $M$. A warped product manifold $N_{1} \times_{f} N_{2}$ is called trivial if its warping function $f$ is constant.

The following lemma is well-known.

Lemma 4.1. [5 Let $M=N_{1} \times{ }_{f} N_{2}$ be a warped product manifold with the warping function $f$, then for any $X, Y \in T\left(N_{1}\right)$ and $Z, W \in T\left(N_{2}\right)$, we have

(i) $\nabla_{X} Y \in T\left(N_{1}\right)$,

(ii) $\nabla_{X} Z=\nabla_{Z} X=X(\ln f) Z$,

(iii) $\nabla_{Z} W=\nabla_{Z}^{N_{2}} W-g(Z, W) \vec{\nabla} \ln f$,

where $\nabla$ and $\nabla^{N_{2}}$ denote the Levi-Civita connections on $M$ and $N_{2}$, respectively and $\vec{\nabla} \ln f$ is the gradient of the function $\ln f$ defined as $g(\vec{\nabla} f, X)=X(f)$.

Remark 4.1. It is important to note that for a warped product $N_{1} \times_{f} N_{2}, N_{1}$ is totally geodesic and $N_{2}$ is totally umbilical in $M$ (c.f., [5, 13]).

In this section, we study pointwise semi-slant warped products $M=N^{T} \times{ }_{f} N^{\theta}$ in an $L C K$-manifold $\tilde{M}$ under the assumption that the Lee vector field $\alpha^{\#}$ is tangent to $M$. Clearly, $C R$-warped products and semi-slant warped product submanifolds are special cases of pointwise semi-slant warped product submanifolds $N^{T} \times_{f} N^{\theta}$ such that the slant function $\theta$ satisfies $\theta=\frac{\pi}{2}$ and $\theta=$ constant, respectively. For simplicity, we denote the tangent spaces of $N^{T}$ and $N^{\theta}$ by $\mathfrak{D}$ and $\mathfrak{D}^{\theta}$, respectively.

Proposition 4.1. For a proper pointwise semi-slant warped product $N^{T} \times{ }_{f} N^{\theta}$ in an LCK-manifold $\tilde{M}$, the Lee vector field $\alpha^{\#}$ is orthogonal to $\mathfrak{D}^{\theta}$.

Proof. For any $X \in \Gamma(\mathfrak{D})$ and $Z \in \Gamma\left(\mathfrak{D}^{\theta}\right)$, we have

$$
g(h(X, Y), F Z)=g(h(X, Y), J Z)=-g\left(J \tilde{\nabla}_{X} Y, Z\right)-g\left(\nabla_{X} Y, J Z\right)
$$

Using the definition of covariant derivative of $J$ and Lemma 4.1(i), we find

$$
\begin{aligned}
g(h(X, Y), F Z) & \left.=g\left(\left(\tilde{\nabla}_{X} J\right) Y\right), Z\right)-g\left(\tilde{\nabla}_{X} J Y, Z\right) \\
& =g(X, Y) g(J \lambda, Z)+g(J X, Y) g(\lambda, Z) .
\end{aligned}
$$

Since $h(X, Y)$ is symmetric with respect to $X$ and $Y$, we find $g(J X, Y) g(\lambda, Z)=0$, which implies $g(\lambda, Z)=0$ for any $Z \in \Gamma\left(\mathfrak{D}^{\theta}\right)$.

Remark 4.2. Proposition 4.1 shows that in our case the Lee vector field $\alpha^{\#}$ is in $\mathfrak{D}$.

Remark 4.3. For a proper CR-product of an $L C K$-manifold, the Lee vector field $\alpha^{\#}$ is normal to $\mathfrak{D}^{\perp}$ (see [7]).

Now, we prove the following useful lemma.

Lemma 4.2. Let $M=N^{T} \times{ }_{f} N^{\theta}$ be a pointwise semi-slant warped product submanifold of an LCK-manifold $\tilde{M}$, where $N^{T}$ and $N^{\theta}$ are holomorphic and proper pointwise slant submanifolds of $\tilde{M}$, respectively and the Lee vector field $\lambda$ is tangent to $M$. Then, we have

(i) $g(h(X, Y), F Z)=0$,

(ii) $g(h(X, Z), F W)=[g(\lambda, J X)-J X(\ln f)] g(Z, W)+[g(\lambda, X)-X(\ln f)] g(Z, P W)$ for any $X, Y \in \Gamma(\mathfrak{D})$ and $Z, W \in \Gamma\left(\mathfrak{D}^{\theta}\right)$. 
Proof. From (4.1) with the symmetry of $h$, we have

$$
g(h(X, Y), F Z)=g(X, Y) g(J \lambda, Z)+g(X, J Y) g(\lambda, Z),
$$

for any $X, Y \in \Gamma(\mathfrak{D})$ and $Z \in \Gamma\left(\mathfrak{D}^{\theta}\right)$. Thus, it follows from (4.1) and (4.2) that

$$
g(h(X, Y), F Z)=g(X, Y) g\left(\beta^{\#}, Z\right) .
$$

Hence, the first part of the lemma follows from above relation by using Proposition 4.1 and the fact that $\lambda$ is tangent to $M$. For the second part, we have

$$
g(h(X, Z), F W)=g(h(X, Z), J W)=-g\left(J\left(\tilde{\nabla}_{Z} X-\nabla_{Z} X\right), W\right),
$$

for any $X \in \Gamma(\mathfrak{D})$ and $Z, W \in \Gamma\left(\mathfrak{D}^{\theta}\right)$. Thus, from the covariant derivative property of $J$, we find

$$
g(h(X, Z), F W)=g\left(\left(\tilde{\nabla}_{Z} J\right) X, W\right)-g\left(\tilde{\nabla}_{Z} J X, W\right)+g\left(J \nabla_{Z} X, W\right) .
$$

Using Lemma 4.1(ii), we get

$$
g(h(X, Z), F W)=g\left(\left(\tilde{\nabla}_{Z} J\right) X, W\right)-J X(\ln f) g(Z, W)+X(\ln f) g(J Z, W) .
$$

Now, using (2.2) we find $g(h(X, Z), F W)=[g(\lambda, J X)-J X(\ln f)] g(Z, W)+[X(\ln f)-g(\lambda, X)] g(P Z, W)$, which proves statement (ii).

The following relations are obtained easily by interchanging $X$ with $J X, Z$ with $P Z$, and $W$ with $P W$ in Lemma 4.2 (ii).

$$
\begin{aligned}
g(h(X, P Z), F W)=[ & J X(\ln f)-g(\lambda, J X)] g(Z, P W) \\
& +\cos ^{2} \theta[g(\lambda, X)-X(\ln f)] g(Z, W), \\
g(h(X, Z), F P W)= & \cos ^{2} \theta[X(\ln f)-g(\lambda, X)] g(Z, W) \\
& +[g(\lambda, J X)-J X(\ln f)] g(Z, P W), \\
g(h(X, P Z), F P W)= & \cos ^{2} \theta[g(\lambda, J X)-J X(\ln f)] g(Z, W) \\
& +\cos ^{2} \theta[g(\lambda, X)-X(\ln f)] g(Z, P W), \\
g(h(J X, Z), F W)=[ & X(\ln f)-g(\lambda, X)] g(Z, W) \\
& +[g(\lambda, J X)-J X(\ln f)] g(Z, P W), \\
g(h(J X, P Z), F W)= & g(\lambda, X)-X(\ln f)] g(Z, P W)+ \\
& \cos ^{2} \theta[g(\lambda, J X)-J X(\ln f)] g(Z, W), \\
g(h(J X, Z), F P W)= & \cos ^{2} \theta[J X(\ln f)-g(\lambda, J X)] g(Z, W) \\
& +[X(\ln f)-g(\lambda, X)] g(Z, P W), \\
g(h(J X, P Z), F P W)= & \cos ^{2} \theta[X(\ln f)-g(\lambda, X)] g(Z, W) \\
& +\cos ^{2} \theta[g(\lambda, J X)-J X(\ln f)] g(Z, P W) .
\end{aligned}
$$

Now, we give the following result for later use. 
Corollary 4.1. Let $M=N^{T} \times{ }_{f} N^{\theta}$ be a nontrivial warped product pointwise semislant submanifold of an LCK-manifold $\tilde{M}$ and Lee field tangent to $M$. Then we have

$$
g(h(X, P Z), F W)=-g(h(X, Z), F P W)
$$

for any $X, Y \in \Gamma(\mathfrak{D})$ and $Z, W \in \Gamma\left(\mathfrak{D}^{\theta}\right)$.

Proof. The proof follows from (4.3) and (4.4).

For a proper pointwise semi-slant warped product $M=N^{T} \times{ }_{f} N^{\theta}$ in an $L C K$ manifold $\tilde{M}$, let $\nu$ denote the invariant subbundle of $T^{\perp} M$ which is the orthogonal complement of $F \mathfrak{D}^{\theta}$ in $T^{\perp} M$ so that

$$
T^{\perp} M=F \mathfrak{D}^{\theta} \oplus \nu .
$$

Theorem 4.1. On a proper pointwise semi-slant warped product $M=N^{T} \times{ }_{f} N^{\theta}$ in an $L C K$-manifold $\tilde{M}$, if $h(X, Z) \in \nu$ for any $X \in \Gamma(\mathfrak{D})$ and $Z \in \Gamma\left(\mathfrak{D}^{\theta}\right)$, then we have $\alpha(X)=X(\ln f)$, where $\alpha$ is the Lee form.

Proof. By virtue of (4.2) (i) and the hypothesis of the theorem, we have

$$
[g(\lambda, J X)-J X(\ln f)] g(Z, W)+[g(\lambda, X)-X(\ln f)] g(Z, P W)=0,
$$

for any $X \in \Gamma(\mathfrak{D})$ and $Z, W \in \Gamma\left(\mathfrak{D}^{\theta}\right)$. Also, from (4.8) and the hypothesis of the theorem, we derive

$$
\cos ^{2} \theta[J X(\ln f)-g(\lambda, J X)] g(Z, W)+[X(\ln f)-g(\lambda, X)] g(Z, P W)=0 .
$$

Hence, it follows from (4.11) and (4.12) that

$$
\sin ^{2} \theta[g(\lambda, J X)-J X(\ln f)] g(Z, W)=0 .
$$

Since $M$ is proper pointwise semi-slant and $g$ is the Riemannian metric, the desired result follows from (4.13).

Corollary 4.2. Let $N^{T} \times{ }_{f} N^{\theta}$ be a mixed totally geodesic pointwise semi-slant warped product in an $L C K$-manifold $\tilde{M}$. Then $\alpha(X)=X(\ln f)$ for any $X \in \Gamma(\mathfrak{D})$.

By using Lemma 4.2, we deduce the following result.

Theorem 4.2. Let $M=N^{T} \times{ }_{f} N^{\theta}$ a proper pointwise semi-slant warped product in an LCK-manifold $\tilde{M}$ and the Lee vector field $\alpha^{\#}$ is tangent to $M$. Then

(i) $g\left(A_{F Z} X, Y\right)=0$.

(ii) $g\left(A_{F Z} J X-A_{F P Z} X, W\right)=\sin ^{2} \theta[X(\ln f)-\alpha(X)] g(Z, W)$

for any $X, Y \in \Gamma(\mathfrak{D})$ and $Z, W \in \Gamma\left(\mathfrak{D}^{\theta}\right)$.

Proof. The first part is nothing but Lemma 4.2 (i). The second part follows from (4.4) and (4.6).

Corollary 4.3. There do not exist a mixed totally geodesic CR-warped product submanifold of the form $N^{T} \times{ }_{f} N^{\perp}$ in a Kaehler manifold $\tilde{M}$.

Proof. Follows from Theorem 4.2 (ii). 


\section{Characterizations theorems}

Now, we provide a characterization of pointwise semi-slant warped products.

Theorem 5.1. Let $M=N^{T} \times{ }_{f} N^{\theta}$ a proper pointwise semi-slant warped product in an LCK-manifold $\tilde{M}$ with its Lee vector field $\alpha^{\#}$ tangent to $M$. Then we have

$$
X(\ln f)=\alpha(X)+\tan \theta X(\theta)
$$

for any $X \in \Gamma(\mathfrak{D})$.

Proof. For any $X \in \Gamma(\mathfrak{D})$ and $Z, W \in \Gamma\left(\mathfrak{D}^{\theta}\right)$, we have

$$
g(h(X, P Z), F W)=g\left(\tilde{\nabla}_{X} P Z, F W\right)=g\left(\tilde{\nabla}_{X} P Z, J W\right)-g\left(\tilde{\nabla}_{X} P Z, P W\right) .
$$

From the covariant derivative property of $J$, we obtain

$$
g\left(A_{F W} P Z, X\right)=g\left(\left(\tilde{\nabla}_{X} J\right) P Z, W\right)-g\left(\tilde{\nabla}_{X} J P Z, W\right)-X(\ln f) \cos ^{2} \theta g(Z, W) .
$$

Using (2.2) and orthogonality of vector fields, we find

$$
\begin{aligned}
g\left(A_{F W} P Z, X\right)= & -g\left(\tilde{\nabla}_{X} P^{2} Z, W\right)-g\left(\tilde{\nabla}_{X} F P Z, W\right)-X(\ln f) \cos ^{2} \theta g(Z, W) \\
= & X(\ln f) \cos ^{2} \theta g(Z, W)-\sin 2 \theta X(\theta) g(Z, W)+g\left(A_{F P Z} X, W\right) \\
& -X(\ln f) \cos ^{2} \theta g(Z, W),
\end{aligned}
$$

which implies that

$$
g\left(A_{F P Z} X, W\right)-g\left(A_{F W} P Z, X\right)=\sin 2 \theta X(\theta) g(Z, W) .
$$

On the other hand, from (4.3) and (4.4), we find

$$
g\left(A_{F P Z} X, W\right)-g\left(A_{F W} P Z, X\right)=2 \cos ^{2} \theta(X(\ln f)-\alpha(X)) g(Z, W) .
$$

Thus, equation (5.1) follows from (5.2) and (5.3).

In order to prove another characterization for pointwise semi-slant warped products, recall the following well-known Hiepko's Theorem.

Theorem 5.2. 26] Let $\mathfrak{D}_{1}$ and $\mathfrak{D}_{2}$ be two orthogonal distribution on a Riemannian manifold $M$. Suppose that both $\mathfrak{D}_{1}$ and $\mathfrak{D}_{2}$ are involutive such that $\mathfrak{D}_{1}$ is a totally geodesic foliation and $\mathfrak{D}_{2}$ is a spherical foliation. Then $M$ is locally isometric to a non-trivial warped product $M_{1} \times_{f} M_{2}$, where $M_{1}$ and $M_{2}$ are integral manifolds of $\mathfrak{D}_{1}$ and $\mathfrak{D}_{2}$, respectively.

Now, we are able to prove another characterization of proper pointwise semi-slant warped product submanifolds of the form $N^{T} \times{ }_{f} N^{\theta}$.

Theorem 5.3. Let $M$ be a proper pointwise semi-slant submanifold with invariant distribution $\mathfrak{D}$ and a proper pointwise slant distribution $\mathfrak{D}^{\theta}$ of an LCK-manifold $\tilde{M}$. Then $M$ is locally a warped product submanifold of the form $N^{T} \times_{f} N^{\theta}$ if and only if

$$
A_{F Z} J X-A_{F P Z} X=\sin ^{2} \theta(X(\mu)-\alpha(X)) Z, \forall X \in \Gamma(\mathfrak{D}), Z \in \Gamma\left(\mathfrak{D}^{\theta}\right),
$$

for some smooth function $\mu$ on $M$ satisfying $W(\mu)=0$ for any $W \in \Gamma\left(\mathfrak{D}^{\theta}\right)$. 
Proof. Let $M=N^{T} \times_{f} N^{\theta}$ be a pointwise semi-slant warped product submanifold of an $L C K$-manifold $\tilde{M}$. Then, by Theorem 4.2(i), we have $g\left(A_{F Z} J X, Y\right)=0$ for any $X, Y \in \Gamma\left(T N^{T}\right)$ and $Z \in \Gamma\left(T N^{\theta}\right)$, i.e., $A_{F Z} J X$ has no components in $T N^{T}$. Also, if we interchange $Z$ by $P Z$ in Theorem 4.2(i), then we get $g\left(A_{F P Z} X, Y\right)=0$, i.e., $A_{F P Z} X$ also has no components $T N^{T}$. Therefore, $A_{F Z} J X-A_{F P Z} X$ lies in $T N^{\theta}$ only. Applying this fact together with Theorem 4.2(ii), we obtain (5.4) with $\mu=\ln f$ and $\alpha(X)=g(\lambda, X)$.

Conversely, suppose that $M$ is a proper pointwise semi-slant submanifold of an $L C K$-manifold $\tilde{M}$ such that (5.4) holds. Then it follows from Lemma 3.1 and the given condition (5.4) that $\sin ^{2} \theta g\left(\nabla_{Y} X, Z\right)=0$ for $X, Y \in \Gamma(\mathfrak{D})$ and $Z \in$ $\Gamma\left(\mathfrak{D}^{\theta}\right)$. Since $M$ is a proper pointwise semi-slant submanifold, $g\left(\nabla_{Y} X, Z\right)=0$ holds. Therefore, the leaves of the distribution $\mathfrak{D}$ are totally geodesic in $M$.

On the other hand, it follows from condition (5.4) and Lemma 3.4 that $\sin ^{2} \theta g([Z, W], X)=0$ holds for any $X \in \Gamma(\mathfrak{D})$ and $Z, W \in \Gamma\left(\mathfrak{D}^{\theta}\right)$. Since $M$ is a proper pointwise semi-slant submanifold, we find $g([Z, W], X)=0$. Thus, the slant distribution $\mathfrak{D}^{\theta}$ is integrable.

Now, let us consider the second fundamental form $h^{\theta}$ of a leaf $N^{\theta}$ of $\mathfrak{D}^{\theta}$ in $M$. Then, for any $Z, W \in \Gamma\left(\mathfrak{D}^{\theta}\right)$ and $X \in \Gamma(\mathfrak{D})$, we have

$$
g\left(h^{\theta}(Z, W), X\right)=g\left(\nabla_{Z} W, X\right)=g\left(\tilde{\nabla}_{Z} W, X\right)=g\left(J \tilde{\nabla}_{Z} W, J X\right) .
$$

Using the covariant derivative property of $J$ and the LCK-structure equation, we have

$$
\begin{aligned}
g\left(h^{\theta}(Z, W), X\right)= & g\left(\tilde{\nabla}_{Z} P W, J X\right)+g\left(\tilde{\nabla}_{Z} F W, J X\right)-g\left(\left(\tilde{\nabla}_{Z} J\right) W, J X\right) \\
= & g\left(\tilde{\nabla}_{Z} P W, J X\right)+g\left(\tilde{\nabla}_{Z} F W, J X\right)-g(J Z, W) g(\lambda, J X) \\
& -g(Z, W) g(\lambda, X) .
\end{aligned}
$$

Again, by using the covariant derivative property of $J$, we find

$$
\begin{aligned}
g\left(h^{\theta}(Z, W), X\right)= & -g\left(\tilde{\nabla}_{Z} J P W, X\right)+g\left(\left(\tilde{\nabla}_{Z} J\right) P W, X\right)-g\left(A_{F W} Z, J X\right) \\
& -g(P Z, W) g(\lambda, J X)-g(Z, W) g(\lambda, X) .
\end{aligned}
$$

Therefore, by (2.6) and (2.2), we derive that

$$
\begin{aligned}
g\left(h^{\theta}(Z, W), X\right)= & -g\left(\tilde{\nabla}_{Z} P^{2} W, X\right)-g\left(\tilde{\nabla}_{Z} F P W, X\right)+g(P Z, P W) g(\lambda, X) \\
& +g(Z, P W) g(J \lambda, X)-g\left(A_{F W} J X, Z\right)-g(P Z, W) g(\lambda, J X) \\
& -g(Z, W) g(\lambda, X) .
\end{aligned}
$$

Then, from (2.8) and (2.9), we obtain

$$
\begin{aligned}
g\left(h^{\theta}(Z, W), X\right)= & \cos ^{2} \theta g\left(\tilde{\nabla}_{Z} W, X\right)+\sin 2 \theta Z(\theta) g(W, X)+g\left(A_{F P W} Z, X\right) \\
& +\cos ^{2} \theta g(\lambda, X) g(Z, W)-g\left(A_{F W} J X, Z\right)-(\lambda, X) g(Z, W) \\
= & \cos ^{2} \theta g\left(\nabla_{Z} W, X\right)+g\left(A_{F P W} X-A_{F W} J X, Z\right) \\
& -\sin ^{2} \theta g(\lambda, X) g(Z, W) .
\end{aligned}
$$

From the condition (5.4), we find $\sin ^{2} \theta g\left(h^{\theta}(Z, W), X\right)=-\sin ^{2} \theta X(\mu) g(Z, W)$. Hence, we conclude from the definition of gradient that $h^{\theta}(Z, W)=-\vec{\nabla} \mu g(Z, W)$, which implies that $N^{\theta}$ is totally umbilical in $M$ with the mean curvature vector given by $H^{\theta}=-\vec{\nabla} \mu$. It is easy to see that the mean curvature vector $H^{\theta}$ is parallel 
to the normal connection $D^{\#}$ of $N^{\theta}$ in $M$. For this, let us consider any $Y \in \Gamma(\mathfrak{D})$ and $Z \in \Gamma\left(\mathfrak{D}^{\theta}\right)$, we find that

$$
\begin{aligned}
g\left(D_{Z}^{\#} \vec{\nabla} \mu, Y\right) & =g\left(\nabla_{Z} \vec{\nabla} \mu, Y\right)=Z g(\vec{\nabla} \mu, Y)-g\left(\vec{\nabla} \mu, \nabla_{Z} Y\right) \\
& =Z(Y(\mu))-g(\vec{\nabla} \mu,[Z, Y])-g\left(\vec{\nabla} \mu, \nabla_{Y} Z\right) \\
& =Z(Y(\mu))-g(\vec{\nabla} \mu, Z Y)+g(\vec{\nabla} \mu, Y Z)+g\left(\nabla_{Y} \vec{\nabla} \mu, Z\right) \\
& =Z(Y(\mu))-Z(Y(\mu))+Y(Z(\mu))=0,
\end{aligned}
$$

since $Z(\mu)=0$, for all $Z \in \mathfrak{D}^{\theta}$ and thus $\nabla_{Y} \vec{\nabla} \mu \in \mathfrak{D}$. This means that the mean curvature of $N^{\theta}$ is parallel. Thus the leaves of $\mathfrak{D}^{\theta}$ are totally umbilical with parallel mean curvature. Consequently, the spherical condition is satisfied. Therefore, it follows from Theorem 5.2 that $M$ is a warped product submanifold $N^{T} \times_{f} N^{\theta}$ with warping function $\mu$. Hence the proof is complete.

\section{Chen type inequality FOR POINTWise SEMI-SLANT WARPED PRODUCtS}

In this section, we provide a sharp estimation for the length of the second fundamental form $h$ of an $m$-dimensional proper pointwise semi-slant warped product $M=N^{T} \times_{f} N^{\theta}$ in a $2 k$-dimensional $L C K$-manifold $\tilde{M}$ such that the Lee vector field $\alpha^{\#}$ is tangent to $N^{T}$. Assume $\operatorname{dim} N^{T}=2 p$ and $\operatorname{dim} N^{\theta}=2 q$ so that we have $m=2 p+2 q$.

Consider an orthonormal frame field $\left\{e_{1}, \cdots, e_{p}, e_{p+1}=J e_{1}, \cdots, e_{2 p}=J e_{p}\right\}$ of distribution $\mathfrak{D}$ and an orthonormal frame $\left\{e_{2 p+1}=e_{1}^{\star}, \cdots, e_{2 p+q}=e_{q}^{\star}, e_{2 p+1+q}=\right.$ $\left.e_{q+1}^{\star}=\sec \theta P e_{1}^{\star}, \cdots, e_{m}=e_{2 q}^{\star}=\sec \theta P e_{q}^{\star}\right\}$ of the distribution $\mathfrak{D}^{\theta}$ on $M$. Then

$$
\begin{aligned}
& \left\{e_{m+1}=\tilde{e}_{1}=\csc \theta F e_{1}^{\star} \cdots, e_{m+q}=\tilde{e}_{q}=\csc \theta F e_{q}^{\star},\right. \\
& \left.e_{m+q+1}=\tilde{e}_{q+1}=\csc \theta \sec \theta F P e_{1}^{\star}, \cdots, e_{m+2 q}=\tilde{e}_{2 q}=\csc \theta \sec \theta F P e_{q}^{\star}\right\}
\end{aligned}
$$

is an orthonormal frame of $F \mathfrak{D}^{\theta}$. Let $\left\{e_{m+2 q+1}=\tilde{e}_{2 q+1}, \cdots, e_{2 k}=\tilde{e}_{2 k-m-2 q}\right\}$ be an orthonormal frames of the invariant normal subbundle $\nu$ of $T^{\perp} M$ (cf. (4.10).

In the following, we will use the above frame fields to obtain a sharp estimation for the squared norm $\|h\|^{2}$ of the second fundamental form $h$ in terms of the gradient of the warping function $f$, Lee vector field $\alpha^{\#}$, and the slant function $\theta$ of the proper pointwise semi-slant warped product submanifold $M$ in the $L C K$-manifold $\tilde{M}$. For simplicity, we denote the tangential component of the Lee vector field $\alpha^{\#}$ of $\tilde{M}$ on the pointwise semi-slant warped product $M=N^{T} \times{ }_{f} N^{\theta}$ by $\alpha_{T M}^{\#}$.

Theorem 6.1. Let $M=N^{T} \times_{f} N^{\theta}$ be a pointwise semi-slant warped product submanifold of a locally conformal Kaehler manifold $\tilde{M}$ such that the Lee vector field is tangent to $M$, where $N^{T}$ and $N^{\theta}$ are holomorphic and proper pointwise slant submanifolds of $\tilde{M}$, respectively. Then

(i) The squared norm of the second fundamental form $h$ of $M$ satisfies

$$
\|h\|^{2} \geq 4 q\left(\csc ^{2} \theta+\cot ^{2} \theta\right)\left\{\|\vec{\nabla}(\ln f)\|^{2}+\left\|\alpha_{T M}^{\#}\right\|^{2}-2 G^{*}\right\}
$$

where $G^{*}=\sum_{i=1}^{2 p} g\left(\vec{\nabla}(\ln f), e_{i}\right) g\left(\alpha^{\#}, e_{i}\right),\left\|\alpha_{T M}^{\#}\right\|^{2}$ is the squared norm of $\alpha_{T M}^{\#}, q=\frac{1}{2} \operatorname{dim} N^{T}, p=\frac{1}{2} \operatorname{dim} N^{\theta}$, and $\vec{\nabla}(\ln f)$ is the gradient of $\ln f$.

(ii) If the equality sign of (6.1) holds identically, then $N^{T}$ is totally geodesic and $N^{\theta}$ is totally umbilical in $\tilde{M}$. Furthermore, $M$ is minimal in $\tilde{M}$. 
Proof. From the definition of $h$, we have

$$
\|h\|^{2}=\sum_{i, j=1}^{m} g\left(h\left(e_{i}, e_{j}\right), h\left(e_{i}, e_{j}\right)\right)=\sum_{r=m+1}^{2 k} \sum_{i, j=1}^{m} g\left(h\left(e_{i}, e_{j}\right), e_{r}\right)^{2} .
$$

Decompose the above relation for $F \mathfrak{D}^{\theta}$ - and $\nu$-components as follows

$$
\|h\|^{2}=\sum_{r=1}^{2 q} \sum_{i, j=1}^{m} g\left(h\left(e_{i}, e_{j}\right), \tilde{e}_{r}\right)^{2}+\sum_{r=2 q+1}^{2 k-m-2 q} \sum_{i, j=1}^{m} g\left(h\left(e_{i}, e_{j}\right), \tilde{e}_{r}\right)^{2} .
$$

By computing $F \mathfrak{D}^{\theta}$-components terms and leaving $\nu$-components positive terms, we find

$$
\begin{aligned}
\|h\|^{2} & \geq \sum_{r=1}^{2 q} \sum_{i, j=1}^{2 p} g\left(h\left(e_{i}, e_{j}\right), \tilde{e}_{r}\right)^{2}+2 \sum_{r=1}^{2 q} \sum_{i=1}^{2 p} \sum_{j=1}^{2 q} g\left(h\left(e_{i}, e_{j}^{\star}\right), \tilde{e}_{r}\right)^{2} \\
& +\sum_{r=1}^{2 q} \sum_{i, j=1}^{2 q} g\left(h\left(e_{i}^{\star}, e_{j}^{\star}\right), \tilde{e}_{r}\right)^{2} .
\end{aligned}
$$

In views of Lemma 4.2(i), the first term in the right hand side of (6.3) is identically zero and there is no relation for the last term $g\left(h\left(\mathfrak{D}^{\theta}, \mathfrak{D}^{\theta}\right), F \mathfrak{D}^{\theta}\right)$. Thus compute just the second term of 6.3.

$$
\|h\|^{2} \geq 2 \sum_{r=1}^{2 q} \sum_{i=1}^{2 p} \sum_{j=1}^{2 q} g\left(h\left(e_{i}, e_{j}^{\star}\right), \tilde{e}_{r}\right)^{2} .
$$

It follows from the frame fields of $\mathfrak{D}, \mathfrak{D}^{\theta}$ and $F \mathfrak{D}^{\theta}$ chosen early in this section that

$$
\begin{aligned}
\|h\|^{2} \geq & 2 \csc ^{2} \theta \sum_{i=1}^{p} \sum_{r, j=1}^{q} g\left(h\left(e_{i}, e_{j}^{\star}\right), F e_{r}^{\star}\right)^{2} \\
& +2 \csc ^{2} \theta \sec ^{4} \theta \sum_{i=1}^{p} \sum_{r, j=1}^{q} g\left(h\left(e_{i}, T e_{j}^{\star}\right), F T e_{r}^{\star}\right)^{2} \\
& +2 \csc ^{2} \theta \sum_{i=1}^{p} \sum_{r, j=1}^{q} g\left(h\left(J e_{i}, e_{j}^{\star}\right), F e_{r}^{\star}\right)^{2} \\
& +2 \csc ^{2} \theta \sec ^{4} \theta \sum_{i=1}^{p} \sum_{r, j=1}^{q} g\left(h\left(J e_{i}, T e_{j}^{\star}\right), F T e_{r}^{\star}\right)^{2} \\
& +2 \csc ^{2} \theta \sec ^{2} \theta \sum_{i=1}^{p} \sum_{r, j=1}^{q} g\left(h\left(e_{i}, T e_{j}^{\star}\right), F e_{r}^{\star}\right)^{2} \\
& +2 \csc ^{2} \theta \sec ^{2} \theta \sum_{i=1}^{p} \sum_{r, j=1}^{q} g\left(h\left(e_{i}, e_{j}^{\star}\right), F T e_{r}^{\star}\right)^{2} \\
& +2 \csc ^{2} \theta \sec ^{2} \theta \sum_{i=1}^{p} \sum_{r, j=1}^{q} g\left(h\left(J e_{i}, T e_{j}^{\star}\right), F e_{r}^{\star}\right)^{2} \\
& +2 \csc ^{2} \theta \sec ^{2} \theta \sum_{i=1}^{p} \sum_{r, j=1}^{q} g\left(h\left(J e_{i}, e_{j}^{\star}\right), F T e_{r}^{\star}\right)^{2} .
\end{aligned}
$$


Thus, we derive from Lemma 4.2(ii) and the relations (4.3)-(4.9) that

$$
\begin{aligned}
\|h\|^{2} \geq & 4 q\left(\csc ^{2} \theta+\cot ^{2} \theta\right) \sum_{i=1}^{p}\left(g\left(\lambda, J e_{i}\right)-J e_{i}(\ln f)\right)^{2} \\
& +4 q\left(\csc ^{2} \theta+\cot ^{2} \theta\right) \sum_{i=1}^{p}\left(g\left(\lambda, e_{i}\right)-e_{i}(\ln f)\right)^{2} \\
= & 4 q\left(\csc ^{2} \theta+\cot ^{2} \theta\right)\left\{\|\vec{\nabla} \ln f\|^{2}+\sum_{i=1}^{p}\left[g\left(\alpha^{\#}, e_{i}\right)^{2}+g\left(\alpha^{\#}, J e_{i}\right)^{2}\right]\right. \\
& \left.-2 \sum_{i=1}^{p}\left[\left(e_{i} \ln f\right) g\left(\alpha^{\#}, e_{i}\right)+\left(J e_{i} \ln f\right) g\left(\alpha^{\#}, J e_{i}\right)\right]\right\} \\
= & 4 q\left(\csc ^{2} \theta+\cot ^{2} \theta\right)\left\{\|\vec{\nabla} \ln f\|^{2}+\sum_{i=1}^{2 p}\left[g\left(\alpha^{\#}, e_{i}\right)^{2}-2\left(e_{i} \ln f\right) g\left(\alpha^{\#}, e_{i}\right)\right]\right\} .
\end{aligned}
$$

Since $\alpha^{\#}$ is in $\mathfrak{D}$ and orthogonal to $\mathfrak{D}^{\theta}$, the the above inequality takes the form

$$
\|h\|^{2} \geq 4 q\left(\csc ^{2} \theta+\cot ^{2} \theta\right)\left\{\|\vec{\nabla} \ln f\|^{2}+\left\|\alpha_{T M}^{\#}\right\|^{2}-2 \sum_{i=1}^{2 p}\left(e_{i} \ln f\right) g\left(\alpha^{\#}, e_{i}\right)\right\}
$$

which is the inequality (6.1). If the equality sign of inequality (6.1) holds, then it follows from the leaving $\nu$-components term in (6.2) that

$$
h(X, Y) \perp \nu, \quad \forall X, Y \in \Gamma(T M) .
$$

Similarly, from the vanishing first term and the leaving third term in the righthand-side of (6.3), we get

$$
h(\mathfrak{D}, \mathfrak{D}) \perp F \mathfrak{D}^{\theta}, \quad h\left(\mathfrak{D}^{\theta}, \mathfrak{D}^{\theta}\right) \perp F \mathfrak{D}^{\theta} .
$$

From (6.4) and (6.5), we conclude that

$$
h(\mathfrak{D}, \mathfrak{D})=\{0\}, \quad h\left(\mathfrak{D}^{\theta}, \mathfrak{D}^{\theta}\right)=\{0\} \text { and } h\left(\mathfrak{D}, \mathfrak{D}^{\theta}\right) \subset F \mathfrak{D}^{\theta} .
$$

If $h^{\theta}$ denotes the second fundamental form of $N^{\theta}$ in $M$, then

$$
g\left(h^{\theta}(Z, W), X\right)=g\left(\nabla_{Z} W, X\right)=-(X(\ln f)) g(Z, W)
$$

for any $X \in \Gamma(\mathfrak{D})$ and $Z, W \in \Gamma\left(\mathfrak{D}^{\theta}\right)$.

Since $N^{T}$ is totally geodesic in $M$ [5, 16, 18, using this fact together with (6.6), we know that $N^{T}$ is totally geodesic in $\tilde{M}$. Also, since $N^{\theta}$ is totally umbilical in $M$ (cf. [5, 16]), it follows from (6.7) with this fact that $N^{\theta}$ is totally umbilical in $\tilde{M}$. Therefore, applying all conditions in (6.6) together with these facts, we conclude that $M$ is minimal in $\tilde{M}$. This completes the proof of the theorem.

\section{Some Applications}

In this section, we provide some applications of our previous results.

If $\theta$ is constant in Theorem 5.1, then the warped product would be semi-slant warped product in a $L C K$-manifold. Such submanifolds have been studied by $\mathrm{H}$. M. Tastan, S. G. Aydin in 35.

For semi-slant warped product submanifold of an $L C K$-manifold $\tilde{M}$, Theorem 5.1 gives the following. 
Theorem 7.1. If $N^{T} \times_{f} N^{\theta}$ is a semi-slant warped product submanifold of an LCK-manifold $\tilde{M}$, then $X(\ln f)=\alpha(X)$ for any $X \in \Gamma(\mathfrak{D})$.

When $\tilde{M}$ is Kaehlerian, i.e. $\alpha^{\#}=0$, Theorem 5.1 also implies the following.

Theorem 7.2. There do not exist any proper semi-slant warped product submanifold of the form $M=N^{T} \times{ }_{f} N^{\theta}$ in a Kaehler manifold $\tilde{M}$.

Theorem 7.2 is the main result (Theorem 3.2) of 31. Therefore, Theorem 5.1 also generalizes Theorem 3.2 of 31 .

If $\theta=\pi / 2$ in Theorem [5.3 then $M$ is a CR-submanifold of an $L C K$-manifold. In this case, Theorem 5.3 implies the following.

Theorem 7.3. A CR-submanifold of an LCK-manifold $\tilde{M}$ with the Lee vector field $\lambda=\alpha^{\#}$ tangent to $M$ is a CR-warped product if and only if the Lee vector field is orthogonal to $\mathfrak{D}^{\perp}$ and the shape operator $A$ satisfies

$$
A_{J Z} X=-(g(J \lambda, X)+J X(\mu)) Z,
$$

for each $X \in \Gamma(\mathfrak{D}), Z \in \Gamma\left(\mathfrak{D}^{\perp}\right)$, and for smooth function $\mu$ on $M$ with $W(\mu)=0$, for each $W \in \Gamma\left(\mathfrak{D}^{\perp}\right)$.

Theorem 7.3 is the main result (Theorem 3.5) of [27]. Hence Theorem 5.3 also generalizes the main result of 27 .

If we put $\alpha^{\#}=0$ and $\theta=\frac{\pi}{2}$ in Theorem 5.3 , the submanifold $M$ in Theorem 5.3 is a CR-submanifold of a Kaehler manifold. Such submanifolds have been studied in 13. A characterization theorem for such manifolds is given by following.

Theorem 7.4. A proper CR-submanifold $M$ of a Kaehler manifold $\tilde{M}$ is locally a CR-warped product if and only if its shape operator A satisfies

$$
A_{J Z} X=-J X(\mu) Z, X \in \Gamma(\mathfrak{D}), Z \in \Gamma\left(\mathfrak{D}^{\perp}\right),
$$

for some function $\mu$ on $M$ satisfying $W(\mu)=0, \forall W \in \Gamma\left(\mathfrak{D}^{\perp}\right)$, where $\mathfrak{D}$ and $\mathfrak{D}^{\perp}$ are the holomorphic and totally real distributions of $M$, respectively.

In fact, this theorem is Theorem 4.2 of [13]. Further, if $\alpha^{\#}=0$ and $\theta$ is a slant function in Theorem 5.3 , then Theorem 5.3 becomes the following characterization theorem (Theorem 5.1) of 32].

Theorem 7.5. Let $M$ be a pointwise semi-slant submanifold of a Kaehler manifold $\tilde{M}$. Then $M$ is locally a non-trivial warped product manifold of the form $M=$ $N^{T} \times{ }_{f} N^{\theta}$ such that $N^{\theta}$ is a proper pointwise slant submanifold and $N^{T}$ is a holomorphic submanifold in $\tilde{M}$ if the following condition is satisfied

$$
A_{F P W} X-A_{F W} J X=-\left(\sin ^{2} \theta\right) X(\mu) W, \forall X \in \Gamma(\mathfrak{D}), W \in \Gamma\left(\mathfrak{D}^{\theta}\right),
$$

where $\mu$ is a function on $M$ such that $Z(\mu)=0$, for every $Z \in \Gamma\left(\mathfrak{D}^{\theta}\right)$.

In fact, in the relation (5.4) of Theorem 5.1 in 32 , the term $\left(1+\cos ^{2} \theta\right)$ should be $\left(1-\cos ^{2} \theta\right)$, i.e., there is a missing term in that theorem.

If $\theta$ is constant on $M$ in Theorem 5.3, then $M$ is a semi-slant submanifold of an $L C K$-manifold. In this case, Theorem 5.3 reduces to the following. 
Theorem 7.6. A semi-slant submanifold $M$ of an $L C K$-manifold $\tilde{M}$ is locally a non-trivial warped product manifold of the form $M=N^{T} \times_{f} N^{\theta}$ such that $N^{\theta}$ is a proper slant submanifold and $N^{T}$ is a holomorphic submanifold in $\tilde{M}$ if and only if the shape operator $A$ of $M$ satisfies

$$
A_{F P Z} X-A_{F Z} J X=\sin ^{2} \theta(g(\lambda, X)-X(\mu)) Z, \forall X \in \Gamma(\mathfrak{D}), Z \in \Gamma\left(\mathfrak{D}^{\theta}\right),
$$

for some smooth function $\mu$ on $M$ such that $W(\mu)=0$, for every $W \in \Gamma\left(\mathfrak{D}^{\theta}\right)$.

Now, we provide the following applications of Theorem 6.1

If the slant function $\theta$ is a constant on $M$ in Theorem 6.1, then the pointwise semi-slant warped product submanifold $M$ reduces to a semi-slant warped product $M=N^{T} \times{ }_{f} N^{\theta}$ in an $L C K$-manifold $\tilde{M}$ such that the Lee vector field is tangent to $M$. In this case, the inequality (6.1) remains the same as

$$
\|h\|^{2} \geq 4 q\left(\csc ^{2} \theta+\cot ^{2} \theta\right)\left\{\mid \vec{\nabla} \ln f\left\|^{2}+\right\| \alpha_{T M}^{\#} \|^{2}-2 G^{*}\right\},
$$

where $G^{*}=\sum_{i=1}^{2 p}\left(e_{i} \ln f\right) g\left(\alpha^{\#}, e_{i}\right)$.

But, if we assume $\theta=\frac{\pi}{2}$ in Theorem 6.1, then the pointwise semi-slant warped product submanifold takes the form $M=N^{T} \times_{f} N^{\perp}$ i.e., $M$ is a CR-warped product, where $N^{T}$ and $N^{\perp}$ are holomorphic and totally real submanifolds of $\tilde{M}$, respectively. In this case, Theorem 6.1 reduces to the following.

Theorem 7.7. Let $M=N^{T} \times{ }_{f} N^{\perp}$ be a CR-warped product submanifold of a locally conformal Kaehler manifold $\tilde{M}$ such that the Lee vector field $\lambda=\alpha^{\#}$ is tangent to $M$. Then

(i) The squared norm of the second fundamental form $h$ of $M$ satisfies

$$
\|h\|^{2} \geq 2 q\left\{\|\vec{\nabla}(\ln f)\|^{2}+\left\|\alpha_{T M}^{\#}\right\|^{2}-2 G^{*}\right\} .
$$

(ii) If the equality sign of inequality (7.5) holds identically, then $N^{T}$ and $N^{\perp}$ are totally geodesic and totally umbilical submanifolds of $\tilde{M}$, respectively. Furthermore, $M$ is minimal in $\tilde{M}$.

Theorem (7.7) is the main result (Theorem 4.2) of [7]. Hence, the main result of [7] is a special case of Theorem 6.1.

A Kaehler manifold is an $L C K$-manifold with $\alpha^{\#}=0$. Thus, Theorem 6.1 implies the following.

Theorem 7.8. Let $M=N^{T} \times_{f} N^{\theta}$ be non-trivial warped product pointwise semislant submanifold in a Kaehler manifold $\tilde{M}$. Then

(i) The squared norm of the second fundamental form of $M$ satisfies

$$
\|h\|^{2} \geq 4 q\left(\csc ^{2} \theta+\cot ^{2} \theta\right)\left\|\vec{\nabla}^{T}(\ln f)\right\|^{2}
$$

where $q=\frac{1}{2} \operatorname{dim} N^{\theta}$.

(ii) If equality sign of inequality (7.6) holds, then $N^{T}$ and $N^{\theta}$ are totally geodesic and totally umbilical submanifolds of $\tilde{M}$, respectively. Furthermore, $M$ is not mixed totally geodesic in $\tilde{M}$. 
Theorem 7.8 is a special case of Theorem 6.1 which is Theorem 5.2. of [32].

If we choose $\alpha^{\#}=0$ and $\theta=\frac{\pi}{2}$, then Theorem 6.1 reduces to Theorem 5.1 of 13, which is exactly the following well-known Chen's inequality for $C R$-warped product submanifolds in Kaehler manifolds.

Theorem 7.9. Let $M=N^{T} \times_{f} N^{\perp}$ be a CR-warped product submanifold in a Kaehler manifold $\tilde{M}$. Then, the squared norm of the second fundamental form $h$ of $M$ satisfies

$$
\|h\|^{2} \geq 2 q\left\|\vec{\nabla}^{T} \ln f\right\|^{2}
$$

where $q=\operatorname{dim} N^{\perp}$ and $\vec{\nabla}(\ln f)$ is gradient of $\ln f$. Furthermore, if the equality sign holds identically in the inequality, then $N^{T}$ and $N^{\theta}$ are totally geodesic and totally umbilical submanifolds of $\tilde{M}$, respectively. Moreover, $M$ is minimal in $\tilde{M}$.

\section{EXAMPLES}

Let $\left(x_{1}, \cdots, x_{n}, y_{1}, \cdots, y_{n}\right)$ be the Cartesian coordinates of a Euclidean $2 n$-space $\mathbb{E}^{2 n}$ equipped with the Euclidean metric $g_{0}$. Then $\mathbb{C}^{n}=\left(\mathbb{E}^{2 n}, J, g_{0}\right)$ is a flat Kaehler manifold endowed with the canonical almost complex structure $J$ given by

$$
J\left(x_{1}, \cdots, x_{n}, y_{1}, \cdots, y_{n}\right)=\left(-y_{1}, \cdots,-y_{n}, x_{1}, \cdots, x_{n}\right) .
$$

The following result can be proved in the same way as Proposition 2.2 of [19].

Proposition 8.1. Let $M=N^{T} \times_{f} N^{\theta}$ be a warped product pointwise semi-slant submanifold of a Kaehler manifold $\tilde{M}$. Then, $M$ is also a warped product pointwise semi-slant submanifold with the same slant function in a LCK-manifold $(\tilde{M}, J, \tilde{g})$ with $\tilde{g}=e^{-f} g$, where $f$ is any smooth function on $\tilde{M}$.

Example 8.1. Let $\mathbb{C}^{3}=\left(\mathbb{E}^{6}, J, g_{0}\right)$ be a flat Kaehler manifold defined above. Consider a 4 -dimensional submanifold $M$ of $\mathbb{C}^{3}$ given by

$$
x_{1}=u, x_{2}=-k s \sin r, x_{3}=g(s), y_{1}=v, y_{2}=k s \cos r, y_{3}=h(s),
$$

where $k$ is a positive number and $(g(s), h(s))$ is a unit speed planar curve. Clearly, the tangent bundle $T M$ of $M$ is spanned by

$$
\begin{aligned}
& X_{1}=\frac{\partial}{\partial u}, \quad X_{2}=\frac{\partial}{\partial v}, \quad X_{3}=\left(-k s \cos r \frac{\partial}{\partial x_{2}}-k s \sin r \frac{\partial}{\partial y_{2}}\right), \\
& X_{4}=\left(-k \sin s \frac{\partial}{\partial x_{2}}+g^{\prime}(s) \frac{\partial}{\partial x_{3}}+k \cos s \frac{\partial}{\partial y_{2}}+h^{\prime}(s) \frac{\partial}{\partial y_{3}}\right) .
\end{aligned}
$$

Further, $M$ is a proper semi-slant submanifold with the holomorphic distribution $\mathfrak{D}=\operatorname{Span}\left\{X_{1}, X_{2}\right\}$ and the slant distribution $\mathfrak{D}^{\theta}=\operatorname{Span}\left\{X_{3}, X_{4}\right\}$ with slant angle given by $\theta=\cos ^{-1}\left(k / \sqrt{1+k^{2}}\right)$. Obviously, both $\mathfrak{D}$ and $\mathfrak{D}^{\theta}$ are integrable and totally geodesic in $M$. Let $N^{T}$ and $N^{\theta}$ be integral manifolds of $\mathfrak{D}$ and $\mathfrak{D}^{\theta}$, respectively. Then the metric $\hat{g}$ on $M=N^{T} \times N^{\theta}$ induced from $\mathbb{C}^{3}$ is given by

$$
\hat{g}=g_{T}+g_{N^{\theta}}, \quad g_{T}=d u^{2}+d v^{2}, \quad g_{N^{\theta}}=k^{2} s^{2} d s^{2}+\left(1+k^{2}\right) d s^{2} .
$$

Let $f=f\left(x_{1}, y_{1}\right)$ be a non-constant smooth function on $\mathbb{C}^{3}$ depending only on coordinates $x_{1}, y_{1}$ and let us consider the Riemannian metric $\tilde{g}=e^{-f} g_{0}$ on $\mathbb{C}^{3}$ 
conformal to the standard metric $g_{0}$. Then $\tilde{M}=\left(\mathbb{E}^{6}, J, \tilde{g}\right)$ is a $G C K$-manifold and the metric on $M$ induced from the $G C K$-manifold is the warped product metric:

$$
g_{M}=g_{N^{T}}+e^{-f} g_{N^{\theta}}, \quad g_{N^{T}}=e^{-f} g_{T} .
$$

Thus, it follows Proposition 8.1 that $\left(M, g_{M}\right)$ is a proper warped product semi-slant submanifold in $\tilde{M}=\left(\mathbb{E}^{6}, J, \tilde{g}\right)$. Now, since $f=f\left(x_{1}, y_{1}\right)$ is a smooth function on $\mathbb{C}^{3}$ depending only on coordinates $x_{1}, y_{1}$, it follows from (8.2) that, restricted to the submanifold $M$, the Lee form of $\tilde{M}$ is given by

$$
\alpha=d f=\frac{\partial f}{\partial u_{1}} d u_{1}+\frac{\partial f}{\partial u_{2}} d u_{2} .
$$

Consequently, it follows from (8.4) and (8.5) that the Lee vector field $\alpha^{\#}$ is tangent to $N^{T}$, and hence it is tangent to $M$.

Example 8.2. Consider a 4-dimensional submanifold $M$ of $\mathbb{C}^{3}$ given by

$$
\begin{aligned}
& x_{1}=\frac{1}{2}\left(u_{1}^{2}+u_{2}^{2}\right), \quad x_{2}=u_{3} \cos u_{4}, \quad x_{3}=u_{3} \sin u_{4}, \\
& y_{1}=\frac{1}{2}\left(u_{1}^{2}-u_{2}^{2}\right), \quad y_{2}=u_{4} \cos u_{3}, \quad y_{3}=u_{4} \sin u_{3},
\end{aligned}
$$

defined on an open subset of $\mathbb{E}^{4}$ with $u_{1}, u_{2} \neq 0, u_{3} u_{4} \neq 1$ and $u_{3}-u_{4} \in\left(0, \frac{\pi}{2}\right)$. Then the tangent bundle $T M$ of $M$ is spanned by

$$
\begin{aligned}
& X_{1}=\frac{1}{\sqrt{2}}\left(\frac{\partial}{\partial x_{1}}+\frac{\partial}{\partial y_{1}}\right), X_{2}=\frac{1}{\sqrt{2}}\left(\frac{\partial}{\partial x_{1}}-\frac{\partial}{\partial y_{1}}\right), \\
& X_{3}=\frac{1}{\sqrt{1+u_{4}^{2}}}\left(\cos u_{4} \frac{\partial}{\partial x_{2}}+\sin u_{4} \frac{\partial}{\partial x_{3}}-u_{4} \sin u_{3} \frac{\partial}{\partial y_{2}}+u_{4} \cos u_{3} \frac{\partial}{\partial y_{3}}\right), \\
& X_{4}=\frac{1}{\sqrt{1+u_{3}^{2}}}\left(-u_{3} \sin u_{4} \frac{\partial}{\partial x_{2}}+u_{3} \cos u_{4} \frac{\partial}{\partial x_{3}}+\cos u_{3} \frac{\partial}{\partial y_{2}}+\sin u_{3} \frac{\partial}{\partial y_{3}}\right) .
\end{aligned}
$$

Then $M$ is a proper pointwise semi-slant submanifold such that the holomorphic distribution is given by $\mathfrak{D}=\operatorname{Span}\left\{X_{1}, X_{2}\right\}$ and the proper pointwise slant distribution is $\mathfrak{D}^{\theta}=\operatorname{Span}\left\{X_{3}, X_{4}\right\}$. It is direct to show that the slant function $\theta$ of $\mathfrak{D}^{\theta}$ satisfies

$$
\cos ^{2} \theta=\frac{\left(u_{3} u_{4}-1\right)^{2} \cos ^{2}\left(u_{3}-u_{4}\right)}{\left(1+u_{3}^{2}\right)\left(1+u_{4}^{2}\right)} .
$$

Clearly, both $\mathfrak{D}$ and $\mathfrak{D}^{\theta}$ are integrable and totally geodesic in $M$. Let $N^{T}$ and $N^{\theta}$ be integral manifolds of $\mathfrak{D}$ and $\mathfrak{D}^{\theta}$, respectively. It is to see that the metric $\hat{g}$ on $M=N^{T} \times N^{\theta}$ induced from $\mathbb{C}^{3}$ is given by

$$
\hat{g}=g_{T}+g_{N^{\theta}},
$$

where

$$
g_{T}=2 u_{1}^{2} d u_{1}^{2}+2 u_{2}^{2} d u_{2}^{2}, \quad g_{N^{\theta}}=\left(1+u_{4}^{2}\right) d u_{3}^{2}+\left(1+u_{3}^{2}\right) d u_{4}^{2} .
$$

Let $f=f\left(x_{1}, y_{1}\right)$ be a non-constant smooth function on $\mathbb{C}^{3}$ depending only on coordinates $x_{1}, y_{1}$ and consider the Riemannian metric $\tilde{g}=e^{-f} g_{0}$ on $\mathbb{C}^{3}$ as in Example 8.1. Then the induced metric on $M$ from $\tilde{M}=\left(\mathbb{E}^{6}, J, \tilde{g}\right)$ is the warped product metric:

$$
g_{M}=g_{N^{T}}+e^{-f} g_{N^{\theta}}, \quad g_{N^{T}}=e^{-f} g_{T} .
$$


Now, it follows Proposition 8.1 that $\left(M, g_{M}\right)$ is a proper warped product pointwise semi-slant submanifold in $\tilde{M}=\left(\mathbb{E}^{6}, J, \tilde{g}\right)$. Now, since $f=f\left(x_{1}, y_{1}\right)$ is a smooth function on $\mathbb{C}^{3}$ depending only on coordinates $x_{1}, y_{1}$, it follows from (8.6) that, restricted to the submanifold $M$, the Lee form of $\tilde{M}$ is given by (8.5). Consequently, it follows from (8.5), (8.8), and (8.9) that the Lee vector field $\alpha^{\#}$ is tangent to $M$.

Remark 8.1. By applying the same method as Example 8.2, we may construct many other examples of proper warped product pointwise semi-slant submanifolds $M$ in $L C K$-manifolds whose Lee vector fields are tangent to $M$.

\section{REFERENCES}

[1] Al-Solamy, F. R., Khan, V.A., Uddin, S.: Geometry of warped product semi-slant submanifolds of nearly Kaehler manifolds. Results. Math. 71(3-4), 783-799 (2016).

[2] Bejancu, A.: CR-submanifolds of a Kaehler manifold I. Amer. Math. Soc. 69, 135-142 (1978).

[3] Bejancu, A.: CR-submanifolds of a Kaehler manifold II. Trans. Amer. Soc. 250, 333-345 (1979).

[4] Bejancu, A.: Geometry of CR-submanifolds. Kluwer Academic Publishers, Dordrecht (1986).

[5] Bishop, R.L., O'Neill, B.: Manifolds of negative curvature. Trans. Amer. Math. Soc. 145, 1-49 (1969).

[6] Blair, D.E., Chen, B.-Y.: On CR-submanifolds of Hermitian manifolds. Israel J. Math. 34, no. 4, 353-363 (1979).

[7] Bonanzinga, V., Matsumoto, K.: Warped product CR-submanifolds in locally conformal Kaehler manifolds. Periodica Math. Hungarica 48, 207-221 (2004).

[8] Chen, B.-Y.: Geometry of submanifolds and its applications. Science University of Tokyo, Tokyo, Japan (1973).

[9] Chen, B.-Y.: CR-submanifolds of a Kaehler manifold I. J. Differential Geometry 16, 305-322 (1981).

[10] Chen, B.-Y.: CR-submanifolds of a Kaehler manifold II. J. Differential Geometry 16, 493-509 (1981).

[11] Chen, B.-Y.: Slant immersions. Bull. Austral. Math. Soc. 41, 135-147 (1990).

[12] Chen, B.-Y.: Geometry of slant submanifolds. Katholieke Universiteit Leuven, Leuven, Belgium (1990).

[13] Chen, B.-Y.: Geometry of warped product CR-submanifolds in Kaehler manifolds. Monatsh. Math. 133, 177-195 (2001).

[14] Chen, B.-Y.: Geometry of warped product CR-submanifolds in Kaehler manifolds II. Monatsh. Math. 134, 103-119 (2001).

[15] Chen, B.-Y.: On isometric minimal immersions from warped products into real space forms. Proc. Edinb. Math. Soc. 45(3), 579-587 (2002).

[16] Chen, B.-Y.: Pseudo-Riemannian geometry, $\delta$-invariants and applications. World Scientific, Hackensack, NJ (2011).

[17] Chen, B.-Y.: Geometry of warped product submanifolds: A survey. J. Adv. Math. Stud. 6(2), 1-43 (2013).

[18] Chen, B.-Y.: Differential geometry of warped product manifolds and submanifolds. World Scientific, Hackensack, NJ (2017).

[19] Chen, B.-Y., Garay, O.J.: Pointwise slant submanifolds in almost Hermitian manifolds. Turk. J. Math. 36, 630-640 (2012).

[20] Chen, B.-Y., Shahid, M.H., Al-Solamy, F.R. (eds.): Complex geometry of slant submanifolds. Springer (2021).

[21] Chen, B.-Y., Shahid, M.H., Al-Solamy, F.R. (eds.): Contact geometry of slant submanifolds. Springer (2021).

[22] Chen, B.-Y., Uddin, S.: Warped product pointwise bi-slant submanifolds of Kaehler manifolds. Publ. Math. Debrecen 92(1-2), 183-199 (2018).

[23] Chen, B.-Y., Uddin, S., Al-Solamy, F.R.: Geometry of pointwise CR-Slant warped products in Kaehler manifolds. Rev. Un. Mat. Argentina 61(2), (2020). 
[24] Dragomir, S., Ornea, L.: Locally Conformal Kaehler Geometry. Birkhauser, Basel (1998).

[25] Etayo, F.: On quasi-slant submanifolds of an almost Hermitian manifold. Publ. Math. Debrecen 53, 217-223 (1998).

[26] Hiepko, S.: Eine inner kennzeichungder verzerrten produkte. Math. Ann. 241, 209-215 (1979).

[27] Jamal, N., Khan, K.A., Khan, V.A.: Generic warped product submanifolds in locally conformal Kaehler manifolds. Acta Math. Sci. 30, 1457-1468 (2010).

[28] Matsumoto, K.: On CR-submanifolds of locally conformal Kaehler manifold. J. Korean Math. Soc. 21, 49-61 (1984).

[29] Matsumoto, K.: Warped product semi-slant submanifolds in locally conformal Kaehler manifolds. Proc. Int. Geom. Cent. 10(2), 8-23 (2017).

[30] Papaghiuc, N.: Semi-slant submanifolds of a Kaehlerian manifold. An. Ştiinţ. Univ. Al. I. Cuza Iaşi Secţ. I a Mat. 40(1), 55-61 (1994).

[31] Sahin, B.: Non-existence of warped product semi-slant submanifolds of Kaehler manifold. Geom. Dedicata 117, 195-202 (2006).

[32] Sahin, B.: Warped product pointwise semi-slant submanifolds of Kaehler manifolds. Port. Math. 70, 252-268 (2013).

[33] Shahid, M.H., Husain, S.I.: Generic submanifolds of locally conformal Kaehler manifolds. Soochow J. Math. 14, 111-117 (1988).

[34] Tastan, H.M., Tripathi, M.M.: Semi-slant submanifolds of a locally conformal Kaehler manifold. An. Ştiinţ. Univ. Al. I. Cuza Iaşi Secţ. I a Mat. (N.S.) 62(1), 337-347 (2016).

[35] Tastan, H.M., Aydin, S.G.: Hemi-slant and semi-slant submanifolds in locally conformal Kaehler manifolds. In: Complex Geometry of Slant Submanifolds, B.-Y. Chen, M.H. Shahid, and F.R. Al-Solamy (eds.), Springer, (2021).

[36] Uddin, S., Al-Solamy, F.R., Khan, K.A.: Geometry of warped product pseudo-slant submanifolds in Kaehler manifolds. An. Ştiinţ. Univ. Al. I. Cuza Iaşi Secţ. I a Mat. (N.S.) 62(3), 927-938 (2016).

[37] Uddin, S., Chen, B.-Y., Al-Solamy, F.R.: Warped product bi-slant immersions in Kaehler manifolds. Mediterr. J. Math. 14(2), Art. 95, 11 pp (2016).

[38] Vaisman, I.: On locally conformal almost Kaehler manifolds. Israel J. Math. 24, 338-351 (1976).

[39] Vaisman, I.: On locally and globally conformal Kaehler manifolds. Trans. Amer. Math. Soc. 262, 533-542 (1980).

B.-Y. Chen: Department of Mathematics, Michigan State University, 619 Red Cedar Road, East Lansing, Michigan 48824-1027, U.S.A.

Email address: chenb@msu.edu

F. Alghamdi: Department of Mathematics, Faculty of Science, Jeddah University, 21589 JedDAH, SAUdi Arabia

Email address: fmalghamdi@uj.edu.sa

S. Uddin: Department of Mathematics, Faculty of Science, King Abdulaziz UniverSity, 21589 Jeddah, Saudi Arabia

Email address: siraj.ch@gmail.com 